\title{
Skin autofluorescence provides additional information to the UK Prospective Diabetes Study (UKPDS) risk score for the estimation of cardiovascular prognosis in type 2 diabetes mellitus
}

\author{
H. L. Lutgers • E. G. Gerrits • R. Graaff • T. P. Links • \\ W. J. Sluiter • R. O. Gans • H. J. Bilo • A. J. Smit
}

Received: 24 October 2008 /Accepted: 30 January 2009/Published online: 10 March 2009

(C) The Author(s) 2009. This article is published with open access at Springerlink.com

\begin{abstract}
Aims/hypothesis The UK Prospective Diabetes Study (UKPDS) risk engine has become a standard for cardiovascular risk assessment in type 2 diabetes mellitus. Skin autofluorescence was recently introduced as an alternative tool for cardiovascular risk assessment in diabetes. We investigated the prognostic value of skin autofluorescence for cardiovascular events in combination with the UKPDS risk engine in a cohort of patients with type 2 diabetes managed in primary care.
\end{abstract}

\footnotetext{
H. L. Lutgers $(\bowtie) \cdot$ R. O. Gans $\cdot$ H. J. Bilo $\cdot$ A. J. Smit

Department of Internal Medicine,

University Medical Center Groningen,

Hanzeplein 1,

9700 RB Groningen, the Netherlands

e-mail: h.1.lutgers@int.umcg.nl

E. G. Gerrits • H. J. Bilo

Diabetes Center, Isala Clinics,

Zwolle, the Netherlands

R. Graaff

Department of Biomedical Engineering,

University Medical Center Groningen,

Groningen, the Netherlands

T. P. Links $\cdot$ W. J. Sluiter

Department of Endocrinology,

University Medical Center Groningen,

Groningen, the Netherlands

T. P. Links $\cdot$ R. O. Gans $\cdot$ H. J. Bilo $\cdot$ A. J. Smit

Department of Medicine, University of Groningen,

Groningen, the Netherlands
}

Methods Clinical, UKPDS risk engine and skin autofluorescence data were obtained at baseline in 2001-2002 in the type 2 diabetes group $(n=973)$. Follow-up data concerning fatal and non-fatal cardiovascular events (primary endpoint) were obtained till 2005. Patients were classified as 'low risk' when their 10 year UKPDS risk score for fatal cardiovascular events was $<10 \%$, and 'high risk' if $>10 \%$. Skin autofluorescence was measured noninvasively with an autofluorescence reader. Skin autofluorescence was classified by the median (i.e. low risk < median, high risk $>$ median).

Results The incidence of cardiovascular events was 119 (44 fatal, 75 non-fatal). In multivariate analysis, skin autofluorescence, age, sex and diabetes duration were predictors for the primary endpoint. Addition of skin autofluorescence information to that from the UKPDS risk engine resulted in re-classification of 55 of 203 patients from the low-risk to the high-risk group. The 10 year cardiovascular event rate was higher in patients with a UKPDS score $>10 \%$ when skin autofluorescence was above the median $(55.8 \%$ vs $38.9 \%)$.

Conclusions/interpretation Skin autofluorescence provides additional information to the UKPDS risk engine which can result in risk re-classification of a substantial number of patients. It furthermore identifies patients who have a particularly high risk for developing cardiovascular events.

Keywords Advanced glycation end-products .

Complications · Fluorescence - Type 2 diabetes .

UKPDS risk score 


\section{Abbreviations \\ AF Autofluorescence \\ CV Cardiovascular \\ UKPDS UK Prospective Diabetes Study}

and mortality in patients with relatively recently diagnosed, and well-controlled, type 2 diabetes managed in primary care.

\section{Methods}

Patients The previously described cohort of 973 patients was used for this follow-up study [7]. In short, the cohort was recruited from participants in the Zwolle Outpatient Diabetes project Integrating Available Care (ZODIAC) study, which investigated the effects of a shared-care project in a primary care population of patients with type 2 diabetes in the eastern part of the Netherlands, starting in 1998 [11]. All known type 2 diabetes patients of 32 general practitioners who received their diabetes support in the primary care setting and additionally visited the diabetes outpatient clinic annually were approached for participation. Patients were included from May 2001 to May 2002. The majority were white (97\%). Patients with cognitive disability or a terminal disease were not included in the ZODIAC study and were, consequently, also ineligible for the present study. This study was approved by the local ethical committee. Written informed consent was obtained from the participants.

Materials and techniques Skin autofluorescence was assessed at baseline using the Autofluorescence Reader (a prototype of the current AGE Reader; DiagnOptics, Groningen, the Netherlands) as described previously [7]. In short, the Autofluorescence Reader illuminates a skin surface of $\sim 4 \mathrm{~cm}^{2}$, guarding against surrounding light, with an excitation light source with peak intensity at $\sim 370 \mathrm{~nm}$. Emission light and reflected excitation light from the skin are measured with a spectrometer in the $300-600 \mathrm{~nm}$ range, using a glass fibre.

Measurements were performed at the volar side of the arm. AF was calculated by dividing the average emitted light intensity per $\mathrm{nm}$ in the range between 420 and $600 \mathrm{~nm}$ by the average excitated light intensity per $\mathrm{nm}$ in the range between 300 and $420 \mathrm{~nm}$. AF was expressed as arbitrary units (a.u.), and multiplied by 100 . Overall AF measurements, and the mean age-corrected AF per measuring month, per examiner and per AFR-system did not differ significantly. Repeated AF measurements taken over a single day in control participants and diabetic patients showed an overall Altman error of 5.03\%. Intra-individual seasonal variance among control participants and diabetic patients showed an Altman error of 5.87\%.

Clinical data The methods of baseline clinical data collection and laboratory assessments have been described in detail elsewhere, including the definition of a history of 
any microvascular disease (retinopathy, neuropathy and/or nephropathy) or any macrovascular disease (coronary heart, cerebrovascular and/or peripheral vascular disease) [7]. Follow-up data, obtained in the period between the date of the baseline autofluorescence measurement and 1 January 2005, were used in the analyses.

UKPDS risk score The UKPDS risk score (10 year nonfatal $\mathrm{CHD}$ risk, 10 year fatal $\mathrm{CHD} /$ stroke risk) was calculated with the UKPDS risk engine (version 2.0, www.dtu.ox.ac.uk, accessed 2 January 2007) using the values of the required variables collected at baseline (single observations).

Events Fatal or non-fatal events were registered from the date of inclusion until January 2005. Fatal events were categorised as: due to $\mathrm{CHD}$, International Classification of Diseases, ninth revision (ICD-9) codes 410-414, cerebrovascular disease 430-438, other CV disease 390-409, 415429, 439-459, sudden death 798-798.9 and non-CV deaths (all other causes). The coded causes of death were combined as total mortality (all codes) and CV mortality (390-459 plus sudden death). The following non-fatal CV events were registered: CHD (myocardial infarction, coronary artery bypass surgery and percutaneous coronary intervention), major cerebrovascular accidents or peripheral vascular disease (amputation, percutaneous transluminal angioplasty, bypass surgery). In the case of multiple nonfatal CV events during the follow-up period, only the first event was considered in the study. All cases and time points of $\mathrm{CV}$ death or events were adjudicated by two independent physicians unaware of the skin AF results, using the hospital and general practitioner records. The primary endpoint was 'any CV event' (fatal plus non-fatal $\mathrm{CV}$ events), the secondary endpoints were all-cause mortality plus non-fatal CV events, and all-cause mortality only.

Statistical analysis The size of the cohort was calculated before the start of the study in 2001 to allow sufficient CV events to occur during the follow-up period to detect a $6 \%$ difference in the proportion of patients having and not having an event (93\% vs 87\%) [7, 12]. The incidence rates of endpoints were calculated per 1000 person-years. Univariate and multivariate Cox regression analyses were performed for the primary endpoint and the secondary endpoints. Possible effect-modification of sex, a history of macrovascular disease, or a history of microvascular disease on the relation between skin AF and endpoints was tested by including interaction terms in regression models.

For the analysis of the possible usefulness of combining skin AF result with the UKPDS risk score, the participants were divided into four groups: group 1, those with a 10 year UKPDS risk for a fatal CV event below $10 \%$ and AF below the median; group 2, those with a 10 year UKPDS risk below $10 \%$ and a skin AF value above the median; group 3, those with a 10 year UKPDS risk for a fatal CV event above $10 \%$ and AF below the median; and group 4, those with a 10 year UKPDS risk above $10 \%$ and a skin AF value above the median.

The cut-off point of the UKPDS risk score was chosen because it is used in the Dutch cardiovascular risk management guidelines 2007 as a treatment-decision cutoff value [13]. The classification of skin AF above and below the median was determined previously as a result of receiver-operating curve (ROC) analyses (non-published data): ROC curves of skin AF expressed as continuous variable and using classification defined by median, tertile and quartile were constructed for the endpoint all-cause mortality. Median skin AF gave the highest value of AUC: 0.63 , 95\% CI $0.58-0.69$. Other values were: $\mathrm{AF}$ on a continuous scale, AUC 0.61 (95\% CI 0.55-0.67); $\mathrm{AF}>$ second tertile, AUC 0.59 (95\% CI 0.52-0.65); and AF in highest quartile, AUC 0.57 (95\% CI 0.51-0.64). Therefore, we used median skin AF in the analysis. For the analysis of the possible value of adding information on skin AF to the UKPDS risk score we performed re-classification analyses, calculated AUCs of two models with and without skin AF $[14,15]$ and constructed Kaplan-Meier curves with the cumulative incidence of events in the four subgroups. Differences between the Kaplan-Meier curves were tested using the logrank test.

A $p$ value $<0.05$ (two-tailed) was considered significant. A 0.025 increment of AUC was considered clinically relevant. This study was not powered to reach statistical significance at an AUC increase of 0.025 , which needs a sample size $>10,000$ patients ( $80 \%$ power, $\alpha=0.05$ ).

\section{Results}

Group characteristics Between 2001 and 2002, 973 patients with type 2 diabetes were studied. Six patients were lost to follow-up. The baseline characteristics of the remaining 967 patients are detailed in Table 1. The mean age was 66 years, mean $\mathrm{HbA}_{1 \mathrm{c}} 7.0 \%$ and mean duration of diabetes was 6.3 years. The median follow-up period was 1,131 days (interquartile range [IQR] 1,019-1,212 days). Forty-two patients died from non-CV causes. There were 119 CV events: 44 CV deaths, and 75 non-fatal CV events, corresponding to an incidence rate of this primary endpoint of 40.1 per 1,000 person-years. A history of CHD was present in 204 patients. In these patients, the incidence of the primary endpoint was 75 per 1,000 person-years at 
Table 1 Baseline characteristics of the 967 participants with type 2 diabetes patients for whom follow-up data were available

\begin{tabular}{ll}
\hline Characteristic & Mean (SD) or \% \\
\hline Age (years) & $66(11)$ \\
Male sex (\%) & 47 \\
Smoking (\%) & 19 \\
BMI (kg/m ${ }^{2}$ ) & $29(5)$ \\
Systolic blood pressure (mmHg) & $146(20)$ \\
Diastolic blood pressure (mmHg) & $81(10)$ \\
Diabetes duration (years) & $4.2(1.6-8.3)^{\mathrm{a}}$ \\
HbA ${ }_{1 \mathrm{c}}(\%)$ & $7.0(1.3)$ \\
Creatinine ( $\mu$ mol/l) & $96(20)$ \\
Creatinine clearance (Cockroft formula) (ml/min) & $76(27)$ \\
Urinary albumin/creatinine ratio (mg/mmol) & $1.47(0.8-4.1)^{\mathrm{a}}$ \\
Total cholesterol (mmol/l) & $5.2(1.0)$ \\
Cholesterol/HDL-cholesterol ratio & $4.3(1.2)$ \\
HDL-cholesterol (mmol/l) & $1.3(0.3)$ \\
LDL-cholesterol (mmol/l) & $2.9(0.9)$ \\
Triacylglycerols (mmol/l) & $2.3(1.3)$ \\
Microvascular disease (\%) & 53 \\
Microalbuminuria & 25 \\
Retinopathy & 20 \\
Neuropathy & 29 \\
Macrovascular disease (\%) & 39 \\
CHD & 21 \\
Cerebrovascular disease & 8 \\
Peripheral vascular disease & 22 \\
Skin autofluorescence (a.u.), mean & $2.79(0.8)$ \\
Skin autofluorescence (a.u.), median & $2.69(2.26-3.19)^{\mathrm{a}}$ \\
UKPDS risk score (\%) & $27.4(16.6-46.9)^{\mathrm{a}}$ \\
\hline & \\
\hline
\end{tabular}

Values are expressed as mean (SD) or \% except where indicated

${ }^{\text {a }}$ Median and interquartile range

a.u., arbitrary units

follow-up compared with 32 per 1,000 person-years in patients without a history of CHD.

Validation of UKPDS risk engine For all participants (including those with a history of CHD), the calculated median UKPDS risk score in 10 years for the primary endpoint was 35\%. The calculated median UKPDS risk score in 3.2 years was $12.3 \%$. This expected CV event rate was not significantly different from the observed rate: $13 \%$ in this study.

Predictors of the primary and secondary endpoints Table 2 shows the univariate HRs for the different endpoints. The first multivariate model including all items of the UKPDS risk engine (except atrial fibrillation and ethnicity) plus $\mathrm{AF}$ showed significant HRs for age, diabetes duration and female sex; skin AF was a significant categorical variable (below or above median) and skin AF as a continuous variable for the primary endpoint was nearly significant (Table 2). Smoking, $\mathrm{HbA}_{1 \mathrm{c}}$, systolic blood pressure and lipid profile were not significant. The second multivariate model consisted of a history of microvascular disease, a history of a macrovascular disease, 10 year UKPDS risk score for total fatal $\mathrm{CV}$ disease above $10 \%$ and $\mathrm{AF}$, and showed significant HRs for UKPDS risk score and for a history of macrovascular disease on all endpoints. Significant HRs were only observed for AF at both secondary endpoints (Table 2). A history of microvascular complications was not significant for any endpoint.

There was no effect modification by sex, history of microvascular complications or history of macrovascular complications in both the univariate and multivariate analyses of effects of skin AF on the primary or secondary end-points.

Additional value of skin AF data Table 3 shows multivariate Cox regression analyses and comparison of AUCs for models with the UKPDS risk score (cut-off point 10\%), with or without the addition of skin AF (cut-off point median) for all three endpoints. In model 1 , for the primary endpoint, the AUC of the model including skin AF increased from 0.581 to 0.618 , which is considered clinically relevant $(>0.025)$, but not significant. For the second secondary endpoint, the AUC increased significantly to 0.667 ( $p=0.03$ ). AUC was highest in model 2 (AUC 0.718 ), which included history of microvascular and macrovascular complications and skin AF. The AUC of this model without skin AF was 0.712, so in model $2 \mathrm{AF}$ made no relevant contribution to the prediction of the primary endpoint.

Table 4 presents the proportion of patients initially classified as having a 10 year risk lower or higher than $10 \%$ who would be re-classified to a higher or lower risk category by having a skin AF lower or higher than the median. It appeared that 55 of 203 persons (27\%) with a calculated 10 year fatal CV risk $<10 \%$ with the UKPDS risk engine had an AF above the median, resulting in reclassification from low to high risk. The calculated 10 year $\mathrm{CV}$ event rate (based on the observed CV events in the first 3.2 years) in this group with low risk according to the UKPDS risk score and high risk according to skin AF is $17.8 \%$, which is $59 \%$ higher than that of the patients with low UKPDS risk score and skin AF below the median $(11.2 \%)$. This difference was not significant $(p=0.5)$. Within the high-risk group (UKPDS risk score $>10 \%$ ), the calculated 10 year $\mathrm{CV}$ event rate was significantly higher $(55.8 \%)$ in patients with skin $\mathrm{AF}>$ median compared with patients with skin AF $<$ median $(38.9 \%), p=0.049$. 
Table 2 Results from the univariate and multivariate Cox regression analyses

\begin{tabular}{|c|c|c|c|c|c|c|}
\hline \multirow[t]{2}{*}{ Variable } & \multicolumn{2}{|c|}{ Primary endpoint } & \multicolumn{2}{|c|}{ First secondary endpoint } & \multicolumn{2}{|c|}{ Second secondary endpoint } \\
\hline & HR & $95 \% \mathrm{CI}$ & $\mathrm{HR}$ & $95 \% \mathrm{CI}$ & HR & $95 \% \mathrm{CI}$ \\
\hline \multicolumn{7}{|l|}{ Univariate analysis } \\
\hline $\operatorname{Age}^{a}$ & $1.38^{\mathrm{b}}$ & $1.18-1.58$ & $1.46^{\mathrm{b}}$ & $1.29-1.64$ & $1.78^{\mathrm{b}}$ & $1.51-2.05$ \\
\hline Female sex & $0.64^{\mathrm{b}}$ & $0.45-0.92$ & $0.69^{\mathrm{b}}$ & $0.51-0.95$ & 0.68 & $0.44-1.03$ \\
\hline Diabetes duration $^{\mathrm{a}}$ & $1.22^{\mathrm{b}}$ & $1.07-1.37$ & $1.21^{\mathrm{b}}$ & $1.08-1.34$ & $1.22^{\mathrm{b}}$ & $1.05-1.40$ \\
\hline Current smoking & 1.16 & $0.75-1.79$ & 1.20 & $0.83-1.73$ & 1.52 & $0.94-2.45$ \\
\hline $\mathrm{BMI}^{\mathrm{a}}$ & 0.94 & $0.76-1.12$ & 0.94 & $0.78-1.09$ & 1.01 & $0.80-1.22$ \\
\hline Hypertension & 0.96 & $0.64-1.43$ & 1.06 & $0.74-1.52$ & 1.24 & $0.75-2.07$ \\
\hline Systolic blood pressure $^{\mathrm{a}}$ & 1.08 & $0.90-1.26$ & 1.10 & $0.95-1.25$ & 1.17 & $0.97-1.38$ \\
\hline Diastolic blood pressure $^{\mathrm{a}}$ & 0.83 & $0.65-1.01$ & 0.80 & $0.64-0.95$ & 0.84 & $0.64-1.06$ \\
\hline $\mathrm{HbA}_{1 \mathrm{c}}^{\mathrm{a}}$ & 1.04 & $0.87-1.23$ & 1.03 & $0.89-1.20$ & 1.03 & $0.83-1.26$ \\
\hline Creatinine $^{\mathrm{a}}$ & $1.26^{\mathrm{b}}$ & $1.12-1.40$ & $1.26^{\mathrm{b}}$ & $1.14-1.38$ & $1.30^{\mathrm{b}}$ & $1.15-1.45$ \\
\hline Total cholesterol $^{\mathrm{a}}$ & 0.96 & $0.80-1.15$ & 0.87 & $0.74-1.02$ & 0.80 & $0.64-1.00$ \\
\hline Chol/HDL ratio ${ }^{a}$ & 1.17 & $0.99-1.37$ & 1.13 & $0.97-1.30$ & 1.04 & $0.84-1.28$ \\
\hline HDL-cholesterol $^{\mathrm{a}}$ & 0.86 & $0.78-1.02$ & $0.85^{\mathrm{b}}$ & $0.78-0.97$ & 0.89 & $0.78-1.10$ \\
\hline LDL-cholesterol $^{\mathrm{a}}$ & 0.97 & $0.81-1.16$ & 0.94 & $0.80-1.10$ & 0.95 & $0.77-1.82$ \\
\hline Skin $\mathrm{AF}>$ median & $1.80^{\mathrm{b}}$ & $1.24-2.62$ & $2.05^{\mathrm{b}}$ & $1.48-2.84$ & $3.13^{\mathrm{b}}$ & $1.93-5.08$ \\
\hline History of peripheral vascular disease & $3.19^{\mathrm{b}}$ & $2.22-4.58$ & $3.01^{\mathrm{b}}$ & $2.21-4.12$ & $3.96^{\mathrm{b}}$ & $2.59-6.06$ \\
\hline History of CHD & $2.48^{\mathrm{b}}$ & $1.71-3.60$ & $2.18^{\mathrm{b}}$ & $1.57-3.01$ & $1.98^{\mathrm{b}}$ & $1.26-3.10$ \\
\hline Albuminuria & $1.76^{\mathrm{b}}$ & $1.21-2.56$ & $1.85^{\mathrm{b}}$ & $1.35-2.55$ & $2.50^{\mathrm{b}}$ & $1.63-3.82$ \\
\hline Neuropathy & $1.49^{\mathrm{b}}$ & $1.03-2.16$ & $1.51^{\mathrm{b}}$ & $1.09-2.07$ & $1.85^{\mathrm{b}}$ & $1.20-2.84$ \\
\hline Retinopathy & 1.23 & $0.81-1.88$ & 1.04 & $0.71-1.53$ & 1.17 & $0.70-1.95$ \\
\hline Microvascular complications & 1.25 & $0.87-1.81$ & 1.33 & $0.97-1.83$ & $2.04^{\mathrm{b}}$ & $1.29-3.24$ \\
\hline Macrovascular complications & $4.33^{\mathrm{b}}$ & $2.91-6.45$ & $3.26^{\mathrm{b}}$ & $2.36-4.52$ & $2.99^{\mathrm{b}}$ & $1.93-4.63$ \\
\hline 10 year CHD risk $k^{a, c}$ & $2.19^{\mathrm{b}}$ & $1.48-3.59$ & $2.32^{\mathrm{b}}$ & $1.63-3.56$ & $3.55^{\mathrm{b}}$ & $2.04-6.84$ \\
\hline 10 year risk of fatal $\mathrm{CV}$ event $\mathrm{t}^{\mathrm{a}, \mathrm{c}}$ & $1.80^{\mathrm{b}}$ & $1.34-2.60$ & $1.90^{\mathrm{b}}$ & $1.46-2.61$ & $2.62^{\mathrm{b}}$ & $1.75-4.25$ \\
\hline 10 year risk of fatal $\mathrm{CV}$ event $>10 \%^{\mathrm{c}}$ & $3.97^{\mathrm{b}}$ & $1.94-8.13$ & $3.57^{\mathrm{b}}$ & $1.98-6.42$ & $4.71^{\mathrm{b}}$ & $1.91-11.63$ \\
\hline \multicolumn{7}{|l|}{ Multivariate analysis } \\
\hline \multicolumn{7}{|l|}{ Model 1} \\
\hline $\mathrm{Age}^{\mathrm{a}}$ & $1.34^{\mathrm{b}}$ & $1.11-1.57$ & $1.41^{\mathrm{b}}$ & $1.21-1.61$ & $1.71^{\mathrm{b}}$ & $1.40-2.02$ \\
\hline Diabetes duration $^{\mathrm{a}}$ & $1.18^{\mathrm{b}}$ & $1.01-1.35$ & $1.15^{\mathrm{b}}$ & $1.01-1.29$ & 1.07 & $0.87-1.27$ \\
\hline Female sex & $0.59^{\mathrm{b}}$ & $0.40-0.86$ & $0.65^{\mathrm{b}}$ & $0.46-0.90$ & $0.59^{\mathrm{b}}$ & $0.37-0.94$ \\
\hline Smoking & 1.18 & $0.75-1.86$ & 1.24 & $0.84-1.82$ & $1.71^{\mathrm{b}}$ & $1.03-2.82$ \\
\hline $\mathrm{HbA}_{1 \mathrm{c}}{ }^{\mathrm{a}}$ & 0.97 & $0.79-1.18$ & 0.98 & $0.82-1.16$ & 1.00 & $0.78-1.26$ \\
\hline Systolic blood pressure $^{\mathrm{a}}$ & 1.02 & $0.82-1.20$ & 1.02 & $0.87-1.18$ & 1.10 & $0.85-1.36$ \\
\hline Total cholesterol $^{\mathrm{a}}$ & 1.06 & $0.88-1.28$ & 0.96 & $0.82-1.14$ & 0.88 & $0.70-1.10$ \\
\hline HDL-cholesterol $^{\mathrm{a}}$ & 0.86 & $0.77-1.04$ & 0.86 & $0.78-1.00$ & 0.94 & $0.80-1.24$ \\
\hline Skin AF & 1.46 & $0.97-2.20$ & $1.57^{\mathrm{b}}$ & $1.10-2.25$ & $2.05^{\mathrm{b}}$ & $1.22-3.45$ \\
\hline \multicolumn{7}{|l|}{ Model 2} \\
\hline Microvascular complications & 0.86 & $0.59-1.26$ & 0.94 & $0.68-1.30$ & 1.40 & $0.88-2.25$ \\
\hline Macrovascular complications & $3.79^{\mathrm{b}}$ & $2.51-5.72$ & $2.74^{\mathrm{b}}$ & $1.96-3.83$ & $2.16^{\mathrm{b}}$ & $1.38-3.40$ \\
\hline 10 year risk for fatal CV event $>10 \%{ }^{\mathrm{c}}$ & $2.79^{\mathrm{b}}$ & $1.34-5.84$ & $2.50^{\mathrm{b}}$ & $1.36-4.58$ & $2.78^{\mathrm{b}}$ & $1.10-7.03$ \\
\hline Skin AF & 1.21 & $0.82-1.79$ & $1.46^{\mathrm{b}}$ & $1.04-2.05$ & $2.18^{\mathrm{b}}$ & $1.32-3.59$ \\
\hline
\end{tabular}

Primary endpoint: fatal + non-fatal CV events; first secondary endpoint: non-fatal CV events + all-cause mortality; second secondary endpoint: allcause mortality

${ }^{\mathrm{a}} \mathrm{HR}$ for continuous variables expressed per SD increase

${ }^{\mathrm{b}}$ Significant HR

${ }^{\mathrm{c}}$ Calculated with the UKPDS risk engine 
Table 3 Multivariate Cox regression analyses and comparison of AUCs for models with the UKPDS risk score above $10 \%$, with or without the addition of skin AF

\begin{tabular}{|c|c|c|c|c|c|c|c|c|c|c|c|c|}
\hline & \multicolumn{4}{|c|}{ Primary endpoint } & \multicolumn{4}{|c|}{ First secondary endpoint } & \multicolumn{4}{|c|}{ Second secondary endpoint } \\
\hline & HR & $95 \% \mathrm{CI}$ & AUC & $p$ value $^{\mathrm{a}}$ & HR & $95 \% \mathrm{CI}$ & AUC & $p$ value & HR & $95 \% \mathrm{CI}$ & AUC & $p$ value \\
\hline \multicolumn{13}{|l|}{ Model 1} \\
\hline 10 year risk for fatal $\mathrm{CV}$ event $>10 \%{ }^{\mathrm{b}}$ & 3.97 & $1.94-8.13$ & 0.581 & & 3.57 & $1.98-6.42$ & 0.581 & & 4.71 & $1.91-11.6$ & 0.583 & \\
\hline 10 year risk for fatal $\mathrm{CV}$ event $>10 \%{ }^{\mathrm{b}}$ & 3.51 & $1.70-7.25$ & & & 3.03 & $1.67-5.49$ & & & 3.56 & $1.43-8.86$ & & \\
\hline Skin $\mathrm{AF}>$ median & 1.52 & $1.05-2.22$ & 0.618 & 0.295 & 1.75 & $1.26-2.44$ & 0.635 & 0.083 & 2.64 & $1.62-4.31$ & 0.667 & 0.031 \\
\hline \multicolumn{13}{|l|}{ Model 2} \\
\hline Microvascular complications & 0.88 & $0.61-1.28$ & & & 0.98 & $0.71-1.36$ & & & 1.53 & $0.96-2.44$ & & \\
\hline Macrovascular complications & 3.92 & $2.61-5.87$ & & & 2.91 & $2.09-4.06$ & & & 2.45 & $1.57-3.83$ & & \\
\hline 10 year risk for fatal CV event $>10 \%{ }^{b}$ & 2.95 & $1.42-6.12$ & 0.712 & & 2.77 & $1.52-5.04$ & 0.682 & & 3.41 & $1.36-8.52$ & 0.689 & \\
\hline Microvascular complications & 0.86 & $0.59-1.26$ & & & 0.94 & $0.68-1.30$ & & & 1.40 & $0.88-2.25$ & & \\
\hline Macrovascular complications & 3.79 & $2.51-5.72$ & & & 2.74 & $1.96-3.83$ & & & 2.16 & $1.38-3.40$ & & \\
\hline 10 year risk for fatal CV event $>10 \%{ }^{b}$ & 2.79 & $1.34-5.84$ & & & 2.50 & $1.36-8.52$ & & & 2.78 & $1.10-7.03$ & & \\
\hline Skin $\mathrm{AF}>$ median & 1.21 & $0.82-1.79$ & 0.718 & 0.857 & 1.46 & $1.04-2.05$ & 0.705 & 0.460 & 2.18 & $1.32-3.59$ & 0.715 & 0.504 \\
\hline
\end{tabular}

Primary endpoint: fatal + non-fatal CV events; first secondary endpoint: non-fatal CV events + all-cause mortality; second secondary endpoint: allcause mortality

${ }^{a}$ Comparing AUCs of models 1 and 2 with and without skin AF

${ }^{\mathrm{b}}$ Calculated with the UKPDS risk engine

Figure 1 shows Kaplan-Meier survival curves for the primary end-point of the four groups. For this endpoint, survival was significantly different between group 4 and all other groups $(1, p<0.001 ; 2, p<0.05 ; 3, p<0.05)$, and between group 3 and group $1(p<0.01)$. Similar differences between groups were found in the analyses for secondary endpoints (survival curves not shown). When participants with a known history of CHD at baseline were excluded from an analysis, similar differences between the same groups were also found for the primary end-point, except that the difference between groups 3 and 4 lost significance $(p=0.053)$.

\section{Discussion}

This study shows that skin autofluorescence is of additional clinical value in the evaluation of risk of fatal and non-fatal $\mathrm{CV}$ events and total mortality in type 2 diabetes mellitus. It is concluded that skin autofluorescence identifies a patient subgroup with an increased event rate within the group of patients at high risk for a fatal CV event ( $>10 \%$ within the next 10 years) calculated with the UKPDS risk engine. The patient group with a UKPDS risk score $>10 \%$ and a skin AF value above the median had a 10 year event rate $(55.8 \%)$ significantly higher than that of the group with
Table 4 Cardiovascular risk re-classification for the primary endpoint (fatal + non-fatal cardiovascular events) in 967 patients with type 2 diabetes from the addition of skin autofluorescence data to the risk predicted by the UKPDS risk engine

\footnotetext{
${ }^{a}$ Observed events during follow-up (approximately 3 years)

${ }^{\mathrm{b}}$ Calculation based on the observed incidence in approximately the first 3 years
}

\begin{tabular}{|c|c|c|c|c|c|}
\hline $\begin{array}{l}10 \text { year risk categories using } \\
\text { the UKPDS risk engine }\end{array}$ & Total & $\mathrm{AF}<$ median & $\mathrm{AF}>$ median & $p$ value & $\begin{array}{l}\text { No. }(\%) \\
\text { re-classified }\end{array}$ \\
\hline \multicolumn{6}{|l|}{$<10 \%$} \\
\hline$n$ & 203 & 148 & 55 & & $55 / 203(27 \%)$ \\
\hline Events, $n(\%)^{\text {a }}$ & $8(3.9)$ & $5(3.4)$ & $3(5.4)$ & & \\
\hline 10 year event rate ${ }^{b}$ & 12.8 & 11.2 & 17.8 & 0.500 & \\
\hline \multicolumn{6}{|l|}{$>10 \%$} \\
\hline$n$ & 764 & 334 & 430 & & \\
\hline Events, $n(\%)$ & $111(14.5)$ & 39 (11.7) & $72(16.7)$ & & \\
\hline 10 year event rate & 47.9 & 38.9 & 55.8 & 0.049 & \\
\hline \multicolumn{6}{|l|}{ All } \\
\hline$n$ & 967 & & & & \\
\hline Events, $n(\%)$ & $119(12.3)$ & & & & \\
\hline 10 year event rate & 40.5 & & & & \\
\hline
\end{tabular}


UKPDS risk score $>10 \%$ and skin AF below the median $(38.9 \%)$. As the majority $(79 \%)$ of this cohort, who were well controlled in primary care with relatively recent onset of type 2 diabetes, has a UKPDS risk score $>10 \%$, it is clinically helpful to have an extra clinical tool to further differentiate risk.

By adding skin AF categories (below and above the median) and re-classifying patients with UKPDS risk scores $<10 \%$, a substantial proportion of patients $(27 \%)$ would be re-classified to a higher-risk group. The incidence of $\mathrm{CV}$ events in patients with a low risk calculated with the UKPDS risk score was 59\% higher if they had an AF above the median than if the AF was below the median. Reclassification of patients from low to high risk by AF seems, therefore, justified and should result in reconsideration of treatment strategies by extending or intensifying treatment in such individuals. Skin autofluorescence should not result in re-classification of patients from high risk (UKPDS risk score $>10 \%$ ) to low risk when skin AF is below the median, because the incidence rate of CV events is high in this group and 'undertreatment' is undesirable.

A history of macrovascular disease and a UKPDS risk score above $10 \%$ were significant predictors in the multivariate Cox regression analysis for all endpoints, and had the highest hazard ratios. Adding skin autofluorescence to UKPDS risk score in model 1 increased the AUC by an increment considered clinically relevant $(>0.025)$; it was not statistically significant for the primary endpoint because of the sample size. For the endpoint 'all-cause mortality', the increase in AUC of the model with AF was higher and

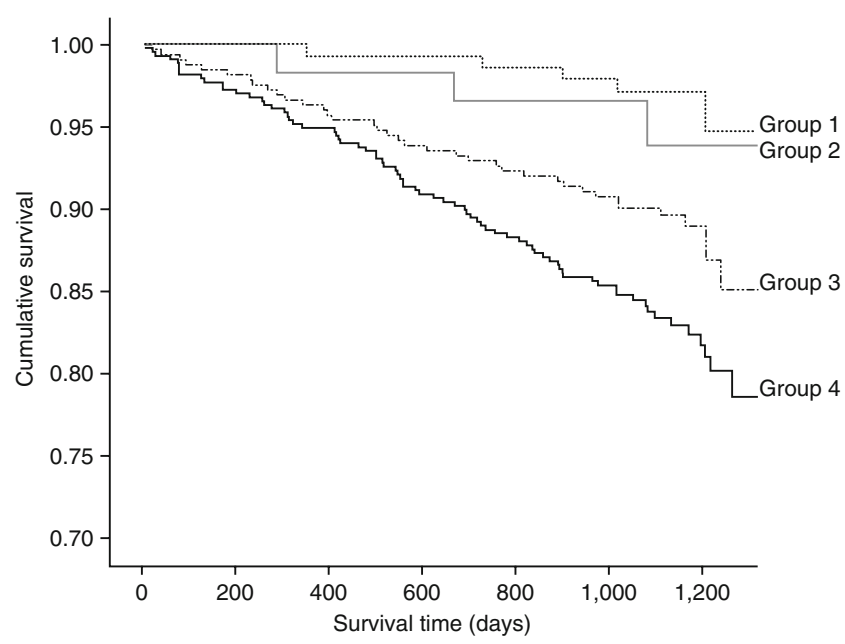

Fig. 1 Kaplan-Meier survival curves for the primary endpoint for the four groups: group 1, AF $<$ median and UKPDS risk score $<10 \%(n=$ $144)$; group $2, \mathrm{AF}>$ median and UKPDS risk score $<10 \%(n=59)$; group 3, AF $<$ median and UKPDS risk score $>10 \%(n=328)$; and group 4, $\mathrm{AF}>$ median and UKPDS risk score $>10 \% \quad(n=436)$. Survival was significantly different between group 4 and all other groups, and between group 3 and group 1 significant. In model 2, where a history of macrovascular disease was also included, the addition of AF was not relevant for the primary endpoint and was just relevant for the secondary endpoint. The small contribution of AF in this model might be due to the relation of AF to macrovascular disease. In our previous study with crosssectional data we showed that AF was related to clinically apparent vascular damage, such as macrovascular disease [7]. The present follow-up study confirms skin AF to be a marker of vascular damage before it becomes clinically apparent because the incidence of CV events has increased in the patient group with a baseline skin AF value above the median.

The contributions of skin AF to all models were higher for the secondary endpoints, where 'all-cause mortality' was included. This suggests that skin AF or AGEs might also be involved in non-CV causes of death such as cancer.

A long-term follow-up study in a Finnish type 2 diabetes mellitus population showed that serum levels of AGEs were predictive for all-cause and CV mortality [16]. Other original papers on the predictive value of AGEs for $\mathrm{CV}$ morbidity or mortality are scarce. However, the mechanisms through which AGEs can result in vascular injury have been described by extension [1, 17, 18]. A recent study showed a pathophysiological example of the role of AGEs in $\mathrm{CV}$ disease by finding increased myocardial AGE deposition in patients with diabetes and heart failure with reduced left ventricular ejection fraction [19].

Despite our inclusion of patients with a history of CHD (which is different from the UKPDS), the expected UKPDS score event rates at 3.2 years in our group were in line with the observed rates, confirming that the UKPDS scores were applicable and calibrated for our group. The incidence of new CV events in our group was twice as high in the $20 \%$ of patients with a history of CHD as in patients without a history of CHD. Including patients with a history of CHD seems to be justified by daily practice where a history of CHD is common even in those newly diagnosed with type 2 diabetes. The UKPDS risk engine was developed in a young group (25-65 years, mean age at diagnosis 52 years) newly diagnosed with type 2 diabetes, without previous CV disease, identified between 1977 and 1991, selected for participation in a clinical trial, and using the mean of $\mathrm{HbA}_{1 \mathrm{c}}$, BP and lipid levels in the first 2 years after diagnosis [9]. Despite the increased incidence at younger ages, type 2 diabetes presents mainly in individuals over 60 years of age in the Netherlands; these patients often have CHD and are already using statins or antihypertensive agents. When blood pressure or cholesterol values from such individuals are entered in the UKPDS risk engine, the calculated risk score might be an underestimated risk. The clinician is expected to identify those at the highest $\mathrm{CV}$ risk 
in order to adapt treatment targets. Our older patient group with $\mathrm{HbA}_{1 \mathrm{c}}$ levels comparable with those of the UKPDS cohort but with prevalent (39\%) macrovascular disease reflects the differences between our 'daily practice' type 2 diabetes cohort and the UKPDS cohort.

In the present study, $\mathrm{HbA}_{1 \mathrm{c}}$ was not found to add predictive value for any of the endpoints. The contribution of $\mathrm{HbA}_{1 \mathrm{c}}$ in the prediction of increased $\mathrm{CV}$ risk in type 2 diabetes was illustrated in the UKPDS by an almost twofold increase in 10 year CHD risk, and by a higher odds ratio for fatal to non-fatal events for both myocardial infarction and stroke $[9,20]$. This seeming discrepancy with our models that include skin AF may reflect the relatively low mean $\mathrm{HbA}_{1 \mathrm{c}}$ in our population. Another potential explanation lies in the following concept. First, skin AF and skin biopsy AGEs may reflect the impact of the history of hyperglycaemic episodes in diabetes and preceding impaired glucose tolerance better than the shortterm measure of hyperglycaemia indicated by $\mathrm{HbA}_{1 \mathrm{c}}$ (weeks). Second, AGEs are also formed during oxidative stress via the pathway of reactive carbonyl compound formation [18]. An earlier study found skin AF to be related to $\mathrm{C}$-reactive protein and inversely related to antioxidant levels, suggesting that skin AF represents inflammatory as well as hyperglycaemic episodes [21]. The DCCT-EDIC group reported that skin collagen AGE and collagen-linked fluorescence from skin biopsies were better predictors than $\mathrm{HbA}_{1 \mathrm{c}}$ of diabetic complications in type 1 diabetes mellitus $[2,3]$. They introduced the idea of 'metabolic memory'that stable AGEs bound to long-lived proteins such as skin collagen provide long-term (years) memory of episodes of hyperglycaemia and oxidative stress - as an explanation for the superiority of skin AGE as a risk predictor. The recent long-term follow-up of glucose control in the UKPDS cohort showed in the intensive therapy group a continued reduction in risk for microvascular complications, myocardial infarction and all-cause mortality during 10 years of post-trial follow-up. This occurred despite the early loss of glycaemic and $\mathrm{HbA}_{1 \mathrm{c}}$ differences, and adds further support to the above concept [22].

Some limitations of our study should be kept in mind: the follow-up period is relatively short. Our calculations were all based on single measurements. The effects of regression dilution bias cannot be excluded. As for the comparison with the UKPDS risk score, in the design of the UKPDS the years $0-4$ were excluded from the analysis, as the mortality rates were lower in the first years of the clinical trial. We used a cohort which had been recruited and followed for at least 3 years before the baseline AF measurements. Furthermore, our population size was small compared with larger studies specifically designed to develop risk-prediction models. This study was not powered to reach significance with the clinically relevant increase in AUC of 0.025 by skin AF. However, relevant increase in AUC became visible in this small cohort at this stage of the follow-up. Finally, our results were obtained in a predominantly white group. AF measurements in darkskinned persons were not considered reliable using the prototype device in our study. Thus, the predictive results of skin AF may not directly be extrapolated to groups with darker skin, but probably need additional validation with newer versions of the AGE Reader.

In conclusion, non-invasive skin $\mathrm{AF}$ is a clinical tool which could be used in addition to the UKPDS risk score to identify diabetic individuals with preclinical vascular damage who have a particularly high risk of developing $\mathrm{CV}$ events. A high skin $\mathrm{AF}$ value can also result in re-classification to a high-risk group patients who are classified as 'low risk' according to the UKPDS risk engine. Although many type 2 diabetes patients are nowadays controlled tightly according to guidelines for their classic risk factors, they still develop micro- and macrovascular complications. Therefore, there is a need for additional markers to identify patients at high risk for complications. Furthermore, risk calculators may underestimate $\mathrm{CV}$ risk in patients already treated with antihypertensive or lipid-lowering drugs. Improvement of risk-prediction strategies is important to further reduce the incidence or progression of complications in patients with diabetes.

Acknowledgement This study was supported by a grant from the Dutch Diabetes Research Foundation (project 2000.00.06).

Duality of interest R. Graaff and A. J. Smit are founders of DiagnOptics, the Netherlands, which manufactures the AGE Reader, based on the prototype used in the study reported here.

Open Access This article is distributed under the terms of the Creative Commons Attribution Noncommercial License which permits any noncommercial use, distribution, and reproduction in any medium, provided the original author(s) and source are credited.

\section{References}

1. Goldin A, Beckman JA, Schmidt AM, Creager MA (2006) Advanced glycation end products. Sparking the development of diabetic vascular injury. Circulation 114:597-605

2. Monnier VM, Bautista O, Kenny D et al (1999) Skin collagen glycation, glycoxidation, and crosslinking are lower in subjects with long-term intensive vs conventional therapy of type 1 diabetes: relevance of glycated collagen products $\mathrm{vs}_{\mathrm{HbA}}$ c as markers of diabetic complications. DCCT Skin Collagen Ancillary Study Group. Diabetes Control and Complications Trial. Diabetes 48:870-880

3. Genuth S, Sun W, Cleary P et al (2005) DCCT Skin Collagen Ancillary Study Group: Glycation and carboxymethyllysine levels in skin collagen predict the risk of future 10-year progression of diabetic retinopathy and nephropathy in the diabetes control and complications trial and epidemiology of diabetes interventions and 
complications participants with type 1 diabetes. Diabetes 54:3103-3111

4. Meerwaldt R, Graaff R, Oomen PH et al (2004) Simple noninvasive assessment of advanced glycation endproduct accumulation. Diabetologia 47:1324-1330

5. Meerwaldt R, Hartog JW, Graaff R et al (2005) Skin autofluorescence, a measure of cumulative metabolic stress and advanced glycation endproducts, predicts mortality in hemodialysis patients. J Am Soc Nephrol 16:3687-3693

6. den Hollander NC, Mulder DJ, Graaff R et al (2007) Advanced glycation end products and the absence of premature atherosclerosis in glycogen storage disease 1a. J Inherit Metab Dis 30:916-923

7. Lutgers HL, Graaff R, Links TP et al (2006) Skin autofluorescence as a non-invasive marker of vascular damage in patients with type 2 diabetes mellitus. Diabetes Care 29:2654-2659

8. Meerwaldt R, Lutgers HL, Links TP et al (2007) Progression of coronary heart disease in diabetes is related to increased skin autofluorescence, a measure of chemical modification by advanced glycoxidation and lipoxidation endproducts and cumulative metabolic burden. Diabetes Care 30:107-112

9. Stevens RJ, Kothari V, Adler AI, Stratton IM, Holman RR, on behalf of the United Kingdom Prospective Diabetes Study (UKPDS) Group (2001) The UKPDS risk engine: a model for the risk of coronary heart disease in Type II diabetes (UKPDS 56). Clin Sci 101:671-679

10. Khot UN, Khot MB, Bajzer CT et al (2003) Prevalence of conventional risk factors in patients with coronary heart disease. JAMA 290:898-904

11. Ubink-Veltmaat LJ, Bilo HJ, Groenier KH, Houweling ST, Rischen RO, Meyboom-de Jong B (2003) Prevalence, incidence and mortality of type 2 diabetes mellitus revisited: a prospective population-based study in the Netherlands (ZODIAC-1). Eur J Epidemiol 18:793-800

12. de Grauw WJ, van de Lisdonk EH, van de Hoogen HJ, van Weel C (1995) Cardiovascular morbidity and mortality in type 2 diabetic patients: a 22-year historical cohort study in Dutch general practice. Diabet Med 12:117-122
13. Burgers JS, Simoons ML, Hoes AW, Stehouwer CD, Stalman WA (2007) Guideline 'Cardiovascular Risk Management'. Ned Tijdschr Geneeskd 151:1068-1074

14. Lloyd-Jones DM, Liu K, Tian L, Greenland P (2006) Narrative review: assessment of $\mathrm{C}$-reactive protein in risk prediction for cardiovascular disease. Ann Int Med 145:35-42

15. Folsom AR, Chambless LE, Duncan BB, Gilbert AC, Pankow JS, The Atherosclerosis Risk in Communities Study Investigators (2003) Prediction of coronary heart disease in middle-aged adults with diabetes. Diabetes Care 26:2777-2784

16. Killhovd BK, Juutilainen A, Lehto et al (2007) Increased serum levels of advanced glycation endproducts predict total, cardiovascular and coronary mortality in women with type 2 diabetes: a population-based 18 year follow-up study. Diabetologia 50:14091417

17. Schmidt AM, Hori O, Brett J, Yan SD, Wautier JL, Stern D (1994) Cellular receptors for advanced glycation end products. Implications for induction of oxidant stress and cellular dysfunction in the pathogenesis of vascular lesions. Arterioscler Thromb 14:1521-1528

18. Brownlee $M$ (2005) The pathobiology of diabetic complications: a unifying mechanism. Diabetes 54:1615-1625

19. van Heerebeek L, Hamdani N, Handoko ML et al (2008) Diastolic stiffness of the failing diabetic heart: importance of fibrosis, advanced glycation end products, and myocyte resting tension. Circulation 117:43-51

20. Stevens RJ, Coleman RL, Adler AI, Stratton IM, Matthews DR, Holman RR (2004) Risk factors for myocardial infarction case fatality and stroke case fatality in type 2 diabetes: UKPDS 66. Diabetes Care 27:201-207

21. Mulder DJ, van de Water T, Lutgers HL et al (2006) Skin autofluorescence, a novel marker for glycation and oxidative stress derived advanced glycation endproducts. An overview of current clinical studies, evidence and limitations. Diabetes Technol Ther 8:523-535

22. Holman RR, Paul SK, Bethel MA, Matthews DR, Neil HA (2008) 10 -year follow-up of intensive glucose control in type 2 diabetes. N Engl J Med 359:1577-1589 